\title{
Effects of Acquisition Parameter Modifications and Field Strength on the Reproducibility of Brain Perfusion Measurements Using Arterial Spin-Labeling
}

\author{
(D) K.P.A. Baas, (D). Petr, (D).P.A. Kuijer, (D)A.J. Nederveen, (D).J.M.M. Mutsaerts, and (D).C.C. van de Ven
}

\begin{abstract}
BACKGROUND AND PURPOSE: Although the added diagnostic value of arterial spin-labeling is shown in various cerebral pathologies, its use in clinical practice is limited. To encourage clinical adoption of ASL, we investigated the reproducibility of CBF measurements and the effects of variations in acquisition parameters compared to the recommended ASL implementation.
\end{abstract}

MATERIALS AND METHODS: Thirty-four volunteers (mean age, $57.8 \pm 17.0$ years; range, 22-80 years) underwent two separate sessions (1.5T and 3T scanners from a single vendor) using a 15-channel head coil. Both sessions contained repeated 3D and 2D pseudocontinuous arterial spin-labeling scans using vendor-recommended acquisition parameters (recommendation paper-based), followed by three 3D pseudocontinuous arterial spin-labeling scans, two with postlabeling delays of 1600 and $2000 \mathrm{~ms}$ and one with increased spatial resolution. All scans were single postlabeling delay. Intrasession (identical acquisitions, scanned five minutes apart) and intersession (first 2D and 3D acquisitions of two sessions) reproducibility was examined as well as the effect of parameter variations on CBF.

RESULTS: Intrasession CBF reproducibility was similar across image readouts and field strengths (within-subject coefficient of variation between $4.0 \%$ and $6.7 \%$ ). Intersession within-subject coefficient of variation ranged from $6.6 \%$ to $14.8 \%$. At $3 \mathrm{~T}$, the $3 \mathrm{D}$ acquisition with a higher spatial resolution resulted in less mixing of GM and WM signal, thus decreasing the bias in GM CBF between the $2 \mathrm{D}$ and $3 \mathrm{D}$ acquisitions $(\triangle \mathrm{CBF}=2.49 \mathrm{~mL} / 100 \mathrm{~g} / \mathrm{min}[P<.001])$. Postlabeling delay variations caused a modest bias $(\Delta \mathrm{CBF}$ between $-3.78[P<.001]$ and $2.83[P<.001] \mathrm{mL} / 100 \mathrm{~g} / \mathrm{min})$.

CONCLUSIONS: Arterial spin-labeling imaging is reproducible at both field strengths, and the reproducibility is not significantly correlated with age. Furthermore, $3 \mathrm{~T}$ tolerates more acquisition parameter variations and allows more extensive optimizations so that $3 \mathrm{D}$ and 2D acquisitions can be compared.

ABBREVIATIONS: ASL = arterial spin-labeling; CoV = coefficient of variation; GraSE = gradient spin-echo; $\mathrm{pCASL}=$ pseudocontinuous arterial spin-labeling; $\mathrm{PLD}=$ postlabeling delay; $\mathrm{PVC}=$ partial volume correction; $\mathrm{WB}=$ whole-brain; $w \mathrm{SCV}=$ within-subject coefficient of variation

A rterial spin-labeling (ASL) MR imaging has the potential to be a cost-effective and safe alternative to contrast agentbased perfusion imaging. ${ }^{1}$ However, despite its proved clinical value, ${ }^{2-4}$ technologic improvements, ${ }^{5-7}$ and consensus recommendation on the implementation, ${ }^{8}$ clinical use of ASL remains limited to date. ${ }^{9}$ ASL is also regularly used in clinical and pharmaceutical trials because in these cases, the preference is to avoid the use of gadolinium-based contrast agents. In such trials, it

Received April 23, 2020; accepted after revision August 17.

From the Department of Radiology and Nuclear Medicine (K.P.A.B., A.J.N.), Amsterdam University Medical Center, Academic Medical Center, University of Amsterdam, Amsterdam, the Netherlands; Institute of Radiopharmaceutical Cancer Research (J.P.), Helmholtz-Zentrum Dresden-Rossendorf, Dresden, Germany; Department of Biomedical Engineering (J.P., H.J.M.M.M.), Institute Hall, Rochester Institute of Technology, Rochester, New York; Department of Radiology and Nuclear Medicine (J.P.A.K., H.J.M.M.M.), Amsterdam University Medical Center, VU University Medical Center, Amsterdam, the Netherlands; Department of Radiology and Nuclear Medicine (H.J.M.M.M.), University Hospital Ghent, Ghent, Belgium; and BIU MR (K.C.C.v.d.V.), Philips Healthcare, Best, the Netherlands. remains a challenge to harmonize imaging protocols over different MR imaging systems, which use different readout types and ASL labeling and imaging parameters. ${ }^{10-12}$ Overcoming these challenges is especially important in multicenter trials as well as in longitudinal studies, in which scanner hardware or software updates and subsequent sequence changes are common.

Several practical limitations hamper the adoption of ASL to image CBF in clinical practice. First, the recommended use of ASL is at a field strength of $3 \mathrm{~T}^{8}{ }^{8}$ However, if ASL is used as an alternative to contrast agent perfusion MR imaging to reduce the

Please address correspondence to Koen P.A. Baas, MSc, Amsterdam University Medical Center, Location AMC, Department of Radiology and Nuclear Medicine, Room Z0-178, Meibergdreef 9, 1105 AZ, the Netherlands; e-mail:

k.p.baas@amsterdamumc.nl

三 Indicates article with supplemental online tables.

Indicates article with supplemental online photo.

http://dx.doi.org/10.3174/ajnr.A6856

AJNR Am J Neuroradiol 42:109-15 Jan 2021 www.ajnr.org 
duration and cost of MR imaging examinations, it would also be preferably conducted at $1.5 \mathrm{~T}$, a field strength that is more widely available. Another limiting factor in clinical practice is that the sensitivity of $\mathrm{CBF}$ values to changes in the acquisition parameters is not well-understood.

Despite the relatively large body of literature on the precision of ASL, in which studies have shown that ASL has a similar reproducibility to $\mathrm{PET}^{13}$ and that whole-brain (WB) reproducibility is comparable among various labeling and readout strategies, ${ }^{10,14,15}$ investigators still question the effects of acquisition parameter changes on the precision of ASL with respect to the recommended implementation as described by the ASL consensus paper. ${ }^{8}$ Other challenges for clinical adoption may be that most ASL reproducibility studies were conducted in young participants and may not be applicable to the elderly population. ${ }^{16}$ Moreover, many studies apply partial volume correction (PVC) to mathematically correct for mixing of GM and WM perfusion, which is inherently present in ASL data due to the relatively large voxels and long readout durations in 3D acquisitions specifically. ${ }^{17}$ Studies are often inconsistent on the corrections applied when reporting CBF values, complicating comparison among studies.

A final challenge for ASL-based perfusion imaging is that standard ASL acquisitions aim to quantify CBF from a single postlabeling delay (PLD) measurement without measuring whether the labeled blood arrived in the tissue. Therefore, it might be unclear whether a low ASL signal is due to decreased perfusion or a delay in arrival. Recently, a novel ASL parameter, which can be derived from single-PLD CBF maps, the spatial coefficient of variation $(\mathrm{CoV})$, was introduced as a proxy of arterial transit time. ${ }^{18}$ Correlation of spatial $\mathrm{CoV}$ with clinical parameters was shown in recent studies, ${ }^{19-21}$ but the reproducibility of this parameter has not yet been reported.

To address the practical issues mentioned above and to encourage further adoption of ASL in clinical practice, this study aims to extend the knowledge on the precision of CBF and spatial CoV measurements. Specifically, this work focuses on studying ASL reproducibility with respect to three common sources of ASL signal variation: 1) age: studying healthy subjects over a large range of adult ages, focusing mostly on older adults because reproducibility studies in this age group are lacking in the literature; 2) field strength and scan parameter variations: assessing the influence of small acquisition parameter variations $^{8}$ at both $3 \mathrm{~T}$ and $1.5 \mathrm{~T}$; and 3) partial volume correction: showing the effect of partial volume correction on deriving pure GM CBF with different scan parameter variations and imaging field strengths.

\section{MATERIALS AND METHODS \\ Participants}

In this study, 34 healthy participants (20 men, 14 women; mean age, $57.8 \pm 17.0$ years) were included. Detailed information about the distribution of participants over different field strengths is given in Online Table 1. This technical study with human participants has been performed under a waiver of institutional review board approval by the Medical Research Ethics Committees United (Nieuwegein, the Netherlands). All participants provided written informed consent and received remuneration for their participation. All experiments were performed in accordance with the Declaration of Helsinki guidelines. Volunteers included in this study participate regularly in MR imaging experiments and are, therefore, trained to lie still for longer periods of time.

On arrival, participants were instructed to refrain from intake of caffeine and smoking during the whole experiment. All participants underwent two scan sessions of 50 minutes with approximately 15 minutes of rest between, during which they were taken out of the scanner. Eight participants were scanned twice at 1.5T, 12 participants were scanned at $1.5 \mathrm{~T}$ and $3 \mathrm{~T}$ in a randomized order, and 14 participants were scanned twice at 3T. To describe the precision of the $\mathrm{CBF}$ and spatial $\mathrm{CoV}$ measurements, we define here intrasession repeatability, considering two within-session repeated measurements, and intersession reproducibility, considering two betweensession repeated measurements. Experiments were performed on the following scanner types: $1.5 \mathrm{~T}$ Ingenia and IngeniaCx and 3T Achieva, Ingenia, and IngeniaCx (Philips Healthcare, Best, the Netherlands). All acquisitions were performed using the standard 15-channel head coil on MR imaging scanner software, Version R5.3 and R5.4, with identical implementation of the ASL sequence. All scanners were in close proximity to one another.

\section{Image Acquisition}

All sessions started with a 3D-T1w scan followed by two identical pseudocontinuous ASL (pCASL) scans with a 3D gradient spinecho (GraSE) readout and two identical pCASL scans with a 2DEPI readout (in an interleaved fashion with an approximately 5minute gap between identical scans) for intrasession repeatability assessment. Next, we acquired two 3D-GraSE pCASL scans with different PLDs: 1600 and 2000 ms. Last, a 3D-GraSE pCASL scan with a higher spatial resolution was acquired (in-plane resolution of $2.75 \times 2.75 \mathrm{~mm}^{2}$ instead of $3.75 \times 3.75 \mathrm{~mm}^{2}$ ). In all pCASL examinations, a labeling duration of $1800 \mathrm{~ms}$ was used, as well as a 4-pulse background-suppression scheme and an integrated M0 scan. Further acquisition parameters are listed in Online Table 2. The initial 3D and 2D ASL scans (sequence numbers 2-5) were obtained from the vendor's imaging data base and in agreement with the consensus recommendation on ASL implementation. ${ }^{8}$

The labeling plane was positioned $9 \mathrm{~cm}$ below the anterior/ posterior commissure plane. A phase-contrast angiography survey scan was performed to check the position of the labeling plane, and if required, the distance was adapted to avoid the labeling plane overlapping the siphons. The full sequence protocol was repeated after a 15-minute break for intersession reproducibility assessment.

\section{Postprocessing}

During reconstruction on the scanner, all ASL scans were quantified according to the single-compartment model recommended in the ASL consensus review. ${ }^{8}$ This includes generation of M0, label, and control images using standard image corrections, including coil sensitivity corrections. Subsequently, a voxel-based calculation was performed to derive the CBF values, as explained in the ASL recommendation paper, using the same assumptions for the T1 of blood (1650 ms at 3T, $1350 \mathrm{~ms}$ at 1.5T), labeling efficiency $(\alpha=0.85)$, and the blood-brain partition coefficient $(\lambda=$ $0.9 \mathrm{~mL} / \mathrm{g}){ }^{8}$ 

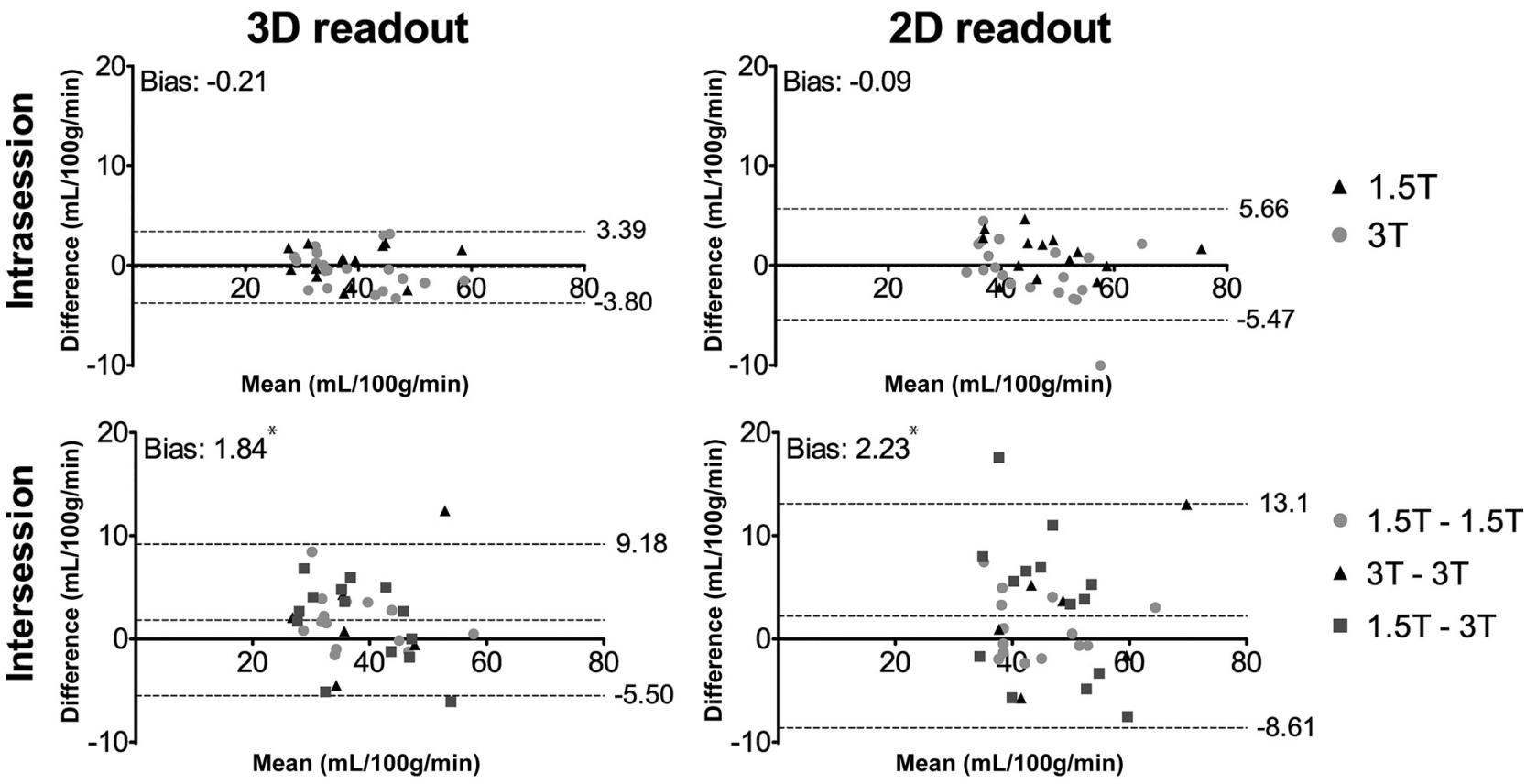

FIG 1. Bland-Altman plots showing agreement on GM CBF between intra- (upper row) and intersession (lower row) repeated 3D $1800 \mathrm{~ms}$ (left column) and 2D $1800 \mathrm{~ms}$ (right column) pCASL scans. The asterisk indicates values significantly different from zero $(P<.05)$.

Further image processing of the CBF maps was performed off-line in ExploreASL (https://sites.google.com/view/exploreasl), which is detailed in depth elsewhere. ${ }^{22}$ Briefly, first, the $3 \mathrm{D}-\mathrm{T} 1 \mathrm{w}$ images were segmented into GM, WM, and CSF and registered to standard space (Montreal Neurological Institute template space) with a voxel size $1.5 \times 1.5 \times 1.5 \mathrm{~mm}^{3}$ using CAT12 (C. Gaser; Structural Brain Mapping Group, Jena University Hospital). ${ }^{23}$ Next, the quantified ASL scans were registered to the 3D-T1w scans by registering the perfusion-weighted maps, the mean label control difference, to the partial GM maps obtained from T1w segmentation using a rigid body registration, with a normalized mutual information criterion. After transforming the ASL images to standard space, the partial GM maps were smoothed to the effective spatial resolution of ASL. The average WB GM CBF was calculated as the average CBF of voxels with $>70 \%$ partial GM content from the partial GM maps in the ASL resolution. WB CBF was calculated by combining the white matter and GM segmentations and thresholding partial GM + partial $\mathrm{WM}>70 \%$. PVC was performed using linear regression on a $5 \times$ 5 kernel as described by Asllani et $\mathrm{al}^{17}$ on GM maps adjusted to the effective spatial resolution to correct for both GM and WM $\mathrm{CBF}$ mixing and for differences in effective spatial resolution. ${ }^{24}$ In this study, we report GM CBF, without PVC, unless otherwise mentioned.

The spatial $\mathrm{CoV}$ was calculated in GM using the CBF maps before PVC: ${ }^{18}$

$$
\text { 1) Spatial } \mathrm{CoV}=\left(S D_{R O I} / M_{\text {ean }}{ }_{R O I}\right) \times 100 \% \text {. }
$$

\section{Statistical Analysis}

To assess the effects of field strength on reproducibility, we divided participants into groups of $1.5 \mathrm{~T}(n=14)$ and $3 \mathrm{~T}(n=20)$ for intrasession and $1.5 \mathrm{~T}-1.5 \mathrm{~T}(n=6), 1.5 \mathrm{~T}-3 \mathrm{~T}(n=14)$, and 3T-3T $(n=14)$ for intersession comparison. For intrasession repeatability, repeated scans of the first scan sessions were used. For intersession reproducibility, the first 3D $1800 \mathrm{~ms}$ and 2D $1800 \mathrm{~ms}$ scans of both sessions were used.

$\mathrm{CBF}$ and spatial CoV data were tested for normality using a Shapiro-Wilk test. Scan types were compared using general linear models; repeated measures ANOVA, with a post hoc Tukey multiple comparison test when comparing normally distributed data, and a Friedman test, with post hoc Dunn multiple comparison test when comparing non-normally distributed data, were included in the analysis.

Repeatability and reproducibility assessment were performed on GM CBF values without PVC. First, the differences in CBF $(\triangle \mathrm{CBF})$ and spatial $\mathrm{CoV}(\Delta$ spatial $\mathrm{CoV})$ between intrasession and intersession repeated measurements were calculated for each participant individually. Intrasession differences were calculated as 3D $1800 \mathrm{~ms}$ run 1 - 3D $1800 \mathrm{~ms}$ run 2 ; intersession differences were calculated as 3D 1800 ms run 1 session 1 - 3D 1800 ms run 1 session 2 . To investigate whether there was a statistically significant correlation between the participants' age and $\triangle \mathrm{CBF}$ and $\Delta$ spatial $\mathrm{CoV}$, we performed a Spearman rank correlation test. Next, as a measure for variability at a group level, the within-subject coefficient of variation (wsCV) was calculated as the ratio of the SD of the differences between the repeated measurements over the mean of the repeated measurements:

$$
\text { 2) } w s C V=100 \% \times\left(S D_{\Delta} \text { Mean }\right) \text {. }
$$

The effect of variations in acquisition parameters was evaluated using the pCASL scans of the first session of all volunteers. First, the mean pair-wise difference, or bias, in observed GM CBF between the recommended 3D acquisitions and acquisitions with 
Table 1: Bias in GM spatial CoV between intra- and intersession repeated 3D and 2D acquisitions ${ }^{\mathrm{a}}$

\begin{tabular}{lll}
\hline & \multicolumn{3}{c}{ GM Spatial CoV } \\
\cline { 2 - 3 } & \multicolumn{1}{c}{ 3D } \\
\hline Intrasession & $-0.001(-0.044,0.042)$ & $-0.004(-0.044,0.035)$ \\
Intersession & $-0.004(-0.085,0.078)$ & $-0.002(-0.125,0.120)$ \\
\hline${ }^{a}$ Numbers in parentheses are 95\% limits of agreement.
\end{tabular}

Table 2: Spearman's $\rho$ and corresponding $P$ values between age and $\triangle C B F$ and between age and $\triangle$ spatial CoV from $3 D$ and $2 D$ ASL scans

\begin{tabular}{cccccc}
\hline & \multicolumn{2}{c}{ 3D } & & \multicolumn{2}{c}{ 2D } \\
\cline { 2 - 3 } \cline { 5 - 6 } \cline { 5 - 6 } & Spearman's $\boldsymbol{\rho}$ & $\boldsymbol{P}$ Value & & Spearman's $\boldsymbol{\rho}$ & $\boldsymbol{P}$ Value \\
\hline$\Delta$ CBF & & & & & \\
Intrasession & -0.18 & .31 & & 0.17 & .34 \\
Intersession & 0.05 & .80 & & 0.05 & .78 \\
$\Delta$ spatial CoV & & & & 0.04 & .82 \\
Intrasession & 0.07 & .71 & & 0.20 & .26 \\
Intersession & 0.15 & .39 & & & \\
\hline
\end{tabular}

Table 3: Intra- and intersession wsCV of GM CBF and GM spatial CoV from 3D $1800 \mathrm{~ms}$ and 2D $1800 \mathrm{~ms}$ scans

\begin{tabular}{lccccc}
\hline & \multicolumn{2}{c}{ GM CBF } & & \multicolumn{2}{c}{ GM Spatial CoV } \\
\cline { 6 - 6 } & 3D & 2D & & 3D & 2D \\
\hline Intrasession & & & & \\
$1.5 T(n=14)$ & $4.4 \%$ & $4.0 \%$ & & $7.6 \%$ & $4.5 \%$ \\
3T $(n=20)$ & $4.7 \%$ & $6.7 \%$ & & $5.1 \%$ & $4.3 \%$ \\
Intersession & & & & \\
$1.5 T-1.5 T(n=6)$ & $13.5 \%$ & $11.7 \%$ & & $6.9 \%$ & $8.4 \%$ \\
$1.5 T-3 \mathrm{~T}(n=14)$ & $10.0 \%$ & $14.8 \%$ & & $15.5 \%$ & $19.1 \%$ \\
$3 \mathrm{~T}-3 \mathrm{~T}(n=14)$ & $6.8 \%$ & $6.6 \%$ & & $11.1 \%$ & $7.6 \%$ \\
\hline
\end{tabular}

parameter variations was calculated by subtracting the CBF value from the images with deviating settings from the recommended $3 \mathrm{D}$ acquisition. To test whether the bias was statistically significantly different from zero, we performed a 1-sample $t$ test. To examine whether the variance across the pair-wise differences between the recommended and deviating ASL acquisitions was significantly different compared with the variance across the pair-wise differences between repeated consensus paper acquisitions, we used the Pitman-Morgan test, a test for equal variance taking repeated measures into account. Statistical significance was defined as $P<.05$.

\section{RESULTS}

Visual inspection showed a large variation in global mean perfusion values among the participants, shown in the Online Figure. Local values of cortical perfusion ranged from $100 \mathrm{~mL} / 100 \mathrm{~g} / \mathrm{min}$ in some participants to relatively low values of about $25 \mathrm{~mL}$ / $100 \mathrm{~g} / \mathrm{min}$ in other participants.

\section{Repeatability and Reproducibility}

First, the GM CBF agreement among repeated, identical, recommendation ASL acquisitions was assessed (Fig 1). For the intrasession repeated scans, the $3 \mathrm{D}$ and $2 \mathrm{D}$ scans showed $95 \%$ limits of agreement of $-3.80,3.39 \mathrm{~mL} / 100 \mathrm{~g} / \mathrm{min}$ and $-5.47,5.66 \mathrm{ml} /$ $100 \mathrm{~g} / \mathrm{min}$, respectively. The intersession repeated scans showed

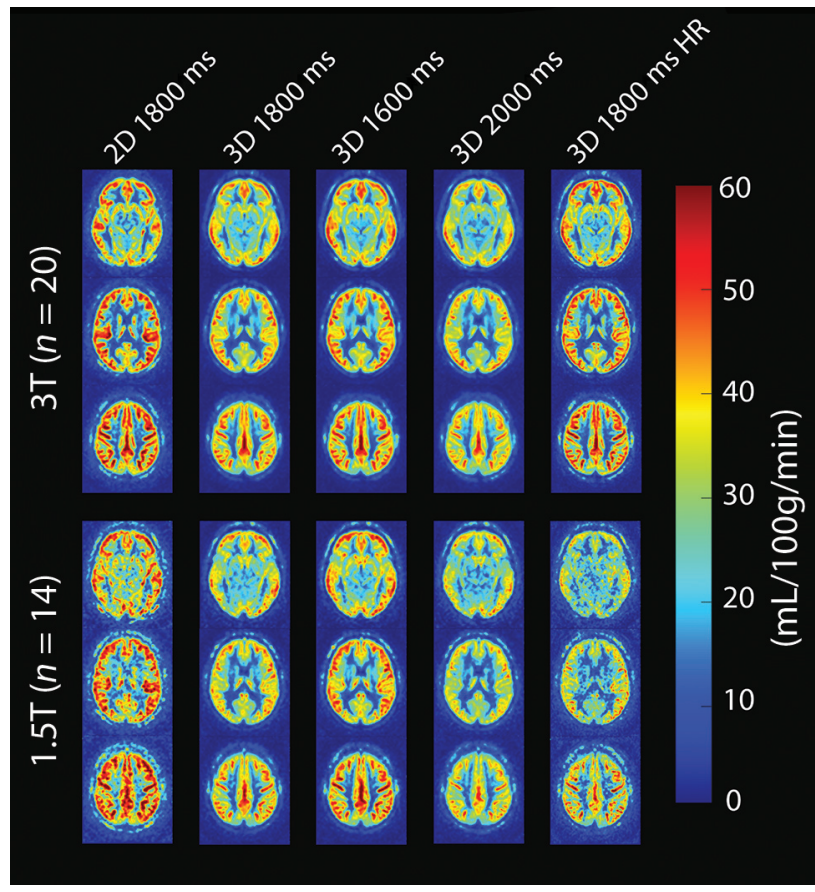

FIG 2. Three axial slices from group-averaged CBF maps of all PCASL acquisitions at both field strengths. Average age of participants scanned at $1.5 \mathrm{~T}$ and $3 \mathrm{~T}$ was $62 \pm 12$ years and $56 \pm 20$ years, respectively. HR indicates high resolution.

$95 \%$ limits of agreement of $-5.50,9.18 \mathrm{~mL} / 100 \mathrm{~g} / \mathrm{min}$ and -8.61 , $13.1 \mathrm{~mL} / 100 \mathrm{~g} / \mathrm{min}$ for $3 \mathrm{D}$ and $2 \mathrm{D}$ scans, respectively.

The bias in GM spatial CoV, together with the 95\% limits of agreement, between repeated $3 \mathrm{D}$ and $2 \mathrm{D}$ scans, was also calculated (Table 1). None of the observed biases in the GM spatial $\mathrm{CoV}$ were significantly different from zero.

No statistically significant correlation between $\triangle \mathrm{CBF}$ and age or between $\Delta$ spatial $\mathrm{CoV}$ and age was observed using the Spearman rank correlation test (Table 2).

The wsCV of GM CBF and spatial $\mathrm{CoV}$ was calculated for both field strengths and all field strength combinations (Table 3). After PVC, the wsCV of GM CBF was similar (data not shown). Intrasession variations were similar at both field strengths. Intersession $\mathrm{CBF}$ variability was lowest when scanning twice at 3T. In contrast, intersession spatial $\mathrm{CoV}$ variability was lowest when scanning twice at $1.5 \mathrm{~T}$.

\section{Scan Parameter Variations}

Averaged ASL scans showed that both 2D $1800 \mathrm{~ms}$ and 3D 1800 $\mathrm{ms}$ high-resolution scans have similar anatomic detail at 3T but had reduced SNR at 1.5T (Fig 2). The high-resolution acquisition at $1.5 \mathrm{~T}$ was excluded from further analysis because the image quality of individual scans was insufficient to perform further analysis. The other 3D-GraSE images were of good quality at both field strengths.

CBF data from several acquisitions showed a non-normal distribution and required nonparametric testing (Online Table 3). A trend of decreasing CBF with increasing PLD was observed (Fig 3). This resulted in statistically significant differences between scans with PLDs of 1600 and $2000 \mathrm{~ms}(P<.05)$. 


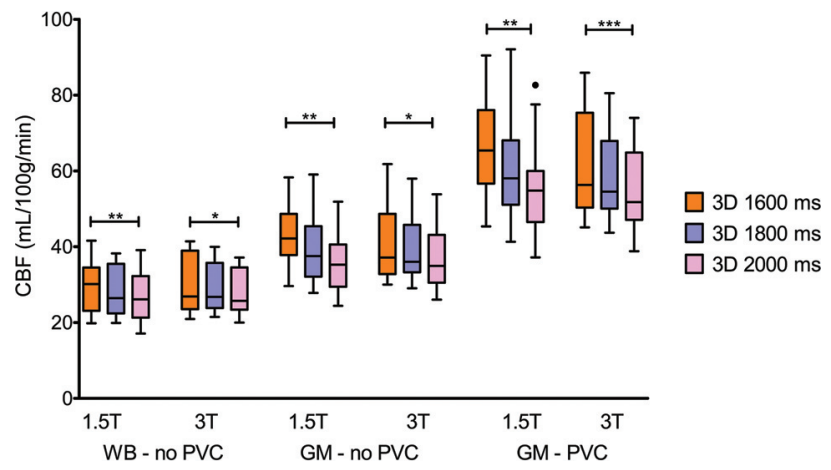

FIG 3. Boxplots showing $W B$ and GM CBF without PVC and GM with PVC per PLD and field strength. One, two, and three asterisks indicate $P$ values $<.05, .01$, and .001 , respectively.

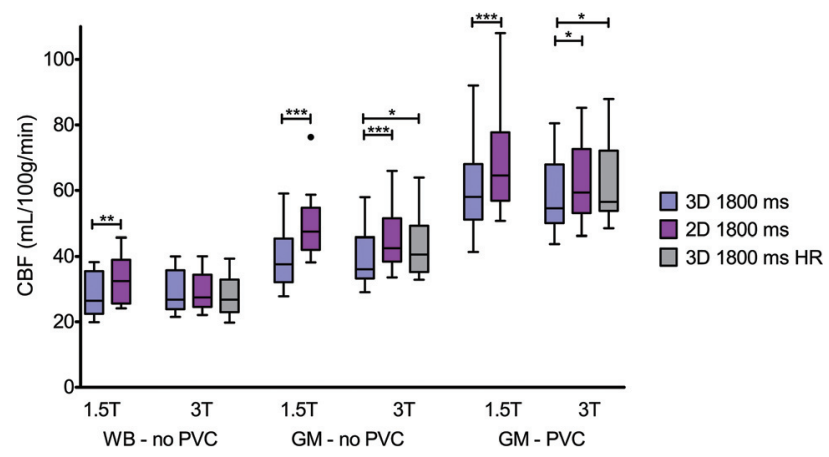

FIG 4. Boxplots showing $W B$ and GM CBF without PVC and GM with PVC, per readout and field strength. One, two, and three asterisks indicate $P$ values $<.05, .01$, and .001 , respectively. HR indicates high resolution.

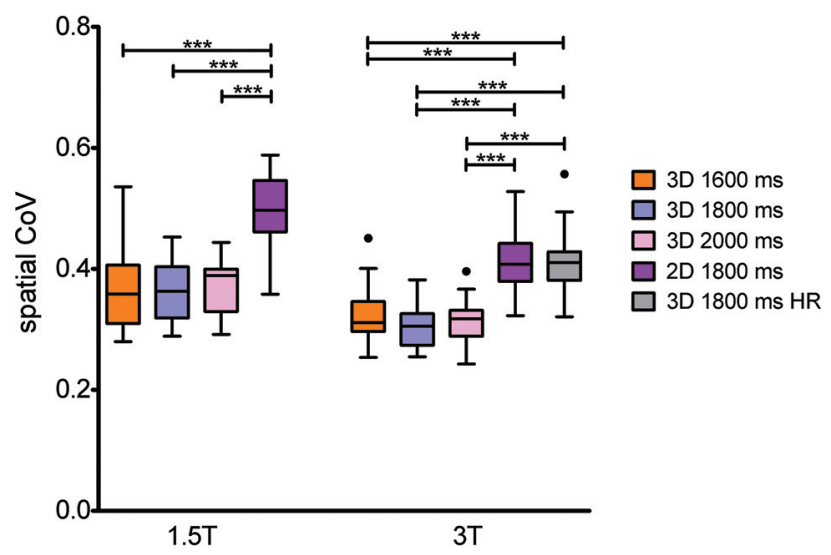

FIG 5. Boxplots showing GM spatial CoV per scan type and field strength. Three asterisks indicate $P$ values $<.001$. HR indicates high resolution.

Statistically significant differences in measured CBF were also observed between acquisitions with different readout types (Fig 4). Similar to Fig 3, the differences observed among scans are decreased when comparing WB instead of GM CBF.

At $1.5 \mathrm{~T}$, the $2 \mathrm{D}$ acquisition resulted in a significantly higher spatial CoV compared with the 3D acquisitions (Fig 5). At 3T, the high-resolution 3D acquisition resulted in significantly higher spatial $\mathrm{CoV}$ values compared with the other $3 \mathrm{D}$ acquisitions as well.
Acquisition parameter variations resulted in statistically significant pair-wise differences in GM CBF $(P<.05)$ (Table 4). Nevertheless, for most combinations, the variance across the observed pair-wise differences was not significantly different compared with the variance across the pair-wise differences between repeated recommendation ASL acquisitions. Moreover, we observed an increase in pair-wise differences among different pCASL acquisitions when scanning at $1.5 \mathrm{~T}$ compared with $3 \mathrm{~T}$. At $3 \mathrm{~T}$, the 2D-EPI acquisition showed the best agreement with the 3D $1800 \mathrm{~ms}$ high-resolution acquisition.

\section{DISCUSSION}

In this study, we have shown that intrasession repeatability and intersession reproducibility of CBF measurements are similar at 3T and $1.5 \mathrm{~T}$ and do not show a statistically significant correlation with age. Additionally, we observed that variations in image readout (2D versus $3 \mathrm{D}$ ) do not have a significant effect on the reproducibility of the CBF measurements. These findings are in agreement with previous reproducibility studies reporting intrasession reproducibility of $3.5 \%-5.5 \%{ }^{14}$ and intersession reproducibility of $10.8 \%-11.3 \%{ }^{10}$ and are comparable in precision with respect to ${ }^{15} \mathrm{O}_{-} \mathrm{H}_{2} \mathrm{O}$ PET. ${ }^{13}$ Results are also in line with studies on reproducibility using different labeling and readout techniques ${ }^{10,14,15,25}$ and the effect of PLD on CBF reproducibility. ${ }^{26}$

Although reproducibility was similar using different pCASL acquisition parameters and different image readouts, the average $\mathrm{CBF}$ values did differ. We observed that differences in measured CBF between $2 \mathrm{D}$ and $3 \mathrm{D}$ readouts were more pronounced in GM compared with WB CBF. This finding could be explained by the difference in effective resolution between $2 \mathrm{D}$ and $3 \mathrm{D}$ readouts. Reduced effective resolution results in more severe partial volume effects and hence affects GM CBF, due to GM and WM CBF mixing, more than WB CBF, in which mixing has a limited effect on the mean. CBF values were also affected by differences in PLD. We suspect that these differences are due to the single-compartment model that was used for quantification, which does not take into account that the duration that the label decays with the tissue T1 differs among the 1600-, 1800-. and 2000-ms PLD sequences. More advanced multicompartment or model-free approaches could account for the T1-decay in blood as well as in tissue. ${ }^{27-29}$ Using an arterial blood $\mathrm{T} 1$ recently determined by $\mathrm{Li}$ et $\mathrm{al},{ }^{30}$ in 2017, we have simulated that a dual compartment model would account for $68 \%$ of the observed difference between the shortest and longest PLD scans (data not shown). The remaining difference could be explained by insufficient delivery of labeled blood in our short PLD data. However, this effect would result in higher spatial $\mathrm{CoV}$, which was not observed in our data.

We have shown that the intrasession repeatability and intersession reproducibility of the spatial CoV, just like CBF, do not show a statistically significant relationship with age. However, spatial $\mathrm{CoV}$ values were higher at $1.5 \mathrm{~T}$ compared with $3 \mathrm{~T}$. This finding could be due to the shorter blood T1, which leads to less signal in distal compared with proximal areas, increasing the spatial CoV. We investigated this effect by calculating the spatial $\mathrm{CoV}$ for each imaging slice individually at both field strengths for a subset of our data. Only at $1.5 \mathrm{~T}$ was a small upward trend in spatial $\mathrm{CoV}$ observed at higher slices (data not shown). This might indicate 
Table 4: Pair-wise differences (mean \pm SD) between observed GM CBF ( $\mathrm{mL} / 100 \mathrm{~g} / \mathrm{min}$ ), without PVC, from different PCASL acquisitions

\begin{tabular}{lccccc}
\hline & 3D 1800 ms & 2D 1800 ms & 3D 1600 ms & 3D 2000 ms & 3D 1800 ms HR \\
\hline 3T & & & & & \\
3D $1800 \mathrm{~ms}$ & $-0.46 \pm 1.88^{\mathrm{a}}$ & $-6.14 \pm 1.45^{\mathrm{b}}$ & $-2.10 \pm 3.20^{\mathrm{bc}}$ & $2.21 \pm 1.85^{\mathrm{b}}$ & $-3.65 \pm 2.00^{\mathrm{b}}$ \\
2D $1800 \mathrm{~ms}$ & & $-0.65 \pm 3.13^{\mathrm{a}}$ & $4.04 \pm 2.95^{\mathrm{bc}}$ & $8.35 \pm 2.12^{\mathrm{b}}$ & $2.49 \pm 2.02^{\mathrm{b}}$ \\
1.5T & & & & \\
3D $1800 \mathrm{~ms}$ & $0.15 \pm 1.77^{\mathrm{a}}$ & $-11.03 \pm 3.21^{\mathrm{bc}}$ & $-3.78 \pm 2.30^{\mathrm{b}}$ & $2.83 \pm 2.06^{\mathrm{b}}$ \\
2D $1800 \mathrm{~ms}$ & & $1.15 \pm 2.04^{\mathrm{a}}$ & $7.25 \pm 4.05^{\mathrm{bc}}$ & $13.86 \pm 3.95^{\mathrm{bc}}$ \\
\hline
\end{tabular}

Note:-HR indicates high-resolution.

${ }^{a}$ Differences between repeated identical PCASL acquisitions with consensus paper parameter settings.

${ }^{\mathrm{b}} \mathrm{A}$ bias that is significantly different from zero $(P<.05)$.

${ }^{\mathrm{C}} \mathrm{A}$ significantly different variance across the observed pair-wise differences between acquisitions compared with the variance across the pair-wise differences between repeated consensus paper parameter settings $(P<.05)$.

that at $1.5 \mathrm{~T}$, distal parts of the brain show a higher spatial CoV due to faster $\mathrm{T} 1$ relaxation at $1.5 \mathrm{~T}$ compared with $3 \mathrm{~T}$.

The higher mean spatial $\mathrm{CoV}$ values subsequently lead to lower intersession wsCV when scanning twice at 1.5T. Moreover, we did observe an increased spatial $\mathrm{CoV}$ in scans with a higher effective resolution, which can be explained by noisier acquisitions and/or higher contrast in these scans. A first investigation of this effect showed that scans with more temporal fluctuations of the ASL signal resulted in higher spatial $\mathrm{CoV}$ values (data not shown). Therefore, we conclude that both CBF and spatial CoV are affected by the effective resolution of the ASL acquisition. While the existing partial volume correction methods for GM CBF can deal with this issue, ${ }^{17,24}$ a similar method that takes into account the differences in GM distribution changes with differences in effective resolution needs to be proposed and validated to be able to account for the resolution-related issues in spatial $\mathrm{CoV}$ calculation.

We observed that scan parameter variations compared with the recommended ASL parameters resulted in significant changes in the observed CBF values. Nevertheless, we consider the observed differences in CBF due to changes in PLD acceptable because the maximum bias between our 3D $1800 \mathrm{~ms}$ and 3D $1600 \mathrm{~ms}$ acquisitions at $1.5 \mathrm{~T}$ was $<10 \%$ of the mean CBF. Moreover, the variance across the pair-wise difference was only once significantly affected for the 3D $1600 \mathrm{~ms}$ acquisition at 3T, indicating that these scan parameter variations only introduce an offset in the measured $\mathrm{CBF}$ but not in a different distribution around the mean $\mathrm{CBF}$ value. Comparing standard $3 \mathrm{D}$ and $2 \mathrm{D}$ ASL acquisitions resulted in a greater bias. At 3T however, the 3D-GraSE acquisition can be optimized to match the effective resolution of the 2D-EPI acquisition. This process results in less mixing of GM and WM signal, reducing the bias in measured CBF. This effect was already hypothesized by Mutsaerts et al, in 2014, based on previous work comparing 3D and 2D ASL scans. ${ }^{10,31,32}$ Overall, the bias between recommended and deviating pCASL acquisitions gets more pronounced at $1.5 \mathrm{~T}$ compared with 3T. This difference could be explained by the decreased SNR and increased loss of label during the PLD, due to the shorter relaxation time of blood at a lower field strength. Therefore, acquisitions at $1.5 \mathrm{~T}$ should be compared with great care and only when the readout type is not changed.

This study has some limitations. Our subjects frequently participate in MR imaging examinations and therefore are trained to lie still for a long time. While this allows us to study the reproducibility of the sequence in the strictly technical sense, the reproducibility in clinical practice might be affected by patient movement. Although motion correction and outlier rejection are typically used by ASL processing software, we expect that motion during the acquisition can still reduce SNR and lead to slight blurring due to imperfect interpolation in the motion correction. This might lead to a decrease of reproducibility, possibly affecting the $3 \mathrm{D}$ sequences more because each average of a $3 \mathrm{D}$ sequence is typically acquired over multiple shots and does not allow simple motion correction as in $2 \mathrm{D}$ readouts. We included relatively few young participants, but because the between-subject variability is usually lower in younger participants and our reproducibility in young volunteers was in agreement with that in previous studies, the effect is probably limited. Furthermore, for practical reasons, not every combination of scanning at $3 \mathrm{~T}$ and $1.5 \mathrm{~T}$ systems was performed in all volunteers, decreasing the available sample size for some combinations. In the scan parameter settings, some small differences such as shot length and through-plane resolution between $1.5 \mathrm{~T}$ and $3 \mathrm{~T}$ sequences remained. Although this might have slightly influenced comparisons between field strenghts, this was necessary to make the sequences comparable while maintaining acceptable image quality.

\section{CONCLUSIONS}

With this work, we provide insights that can help ASL acquisition parameter optimization in the setting of clinical practice as well as in clinical trials in which MR imaging systems with different ASL applications are used. Our data show that ASL imaging is well reproducible at $3 \mathrm{~T}$ and $1.5 \mathrm{~T}$ and that the differences between repeated measurements show no statistically significant correlation with age. It should be noted that, Scanning at 3T offers more tolerance for scan parameter variations compared with $1.5 \mathrm{~T}$ and allows more extensive acquisition parameter optimization, resulting in good agreement among ASL acquisitions. We advise that clinical comparisons at $1.5 \mathrm{~T}$ should be made only on the basis of scans with identical acquisitions settings. With these precautions taken into account, our findings advocate the use of ASL as a cost-effective and safe alternative to contrast agent-based perfusion at both field strengths.

\section{ACKNOWLEDGMENTS}

The authors thank Professor M.J.P. van Osch for the useful discussions and proofreading of this work. 
17. Asllani I, Borogovac A, Brown TR. Regression algorithm correcting for partial volume effects in arterial spin labeling MRI. Magn Reson Med 2008;60:1362-71 CrossRef Medline

\section{REFERENCES}

1. Detre JA, Leigh JS, Williams DS, et al. Perfusion imaging. Magn Reson Med 1992;23:37-45 CrossRef Medline

2. Telischak NA, Detre JA, Zaharchuk G. Arterial spin labeling MRI: clinical applications in the brain. J Magn Reson Imaging 2015;41:1165-80 CrossRef Medline

3. Detre JA, Rao H, Wang DJ, et al. Applications of arterial spin labeled MRI in the brain. J Magn Reson Imaging 2012;35:1026-37 CrossRef Medline

4. Hendrikse J, Petersen ET, Golay X. Vascular disorders: insights from arterial spin labeling. Neuroimaging Clin N Am 2012;22:25969 CrossRef Medline

5. Garcia DM, Duhamel G, Alsop DC. Efficiency of inversion pulses for background suppressed arterial spin labeling. Magn Reson Med 2005;54:366-72 CrossRef Medline

6. Ye FQ, Frank JA, Weinberger DR, et al. Noise reduction in 3D perfusion imaging by attenuating the static signal in arterial spin tagging (ASSIST). Magn Reson Med 2000;44:92-100 CrossRef Medline

7. Günther M, Oshio K, Feinberg DA. Single-shot 3D imaging techniques improve arterial spin labeling perfusion measurements. Magn Reson Med 2005;54:491-98 CrossRef Medline

8. Alsop DC, Detre JA, Golay X, et al. Recommended implementation of arterial spin-labeled perfusion MRI for clinical applications: a consensus of the ISMRM Perfusion Study group and the European Consortium for ASL in Dementia. Magn Reson Med 2015;73:10216 CrossRef Medline

9. Keil VC, Smits M, Thust $S$, et al. The long road from invention to implementation: a PAN-European neuroradiological survey on quantitative MRI techniques in clinical practice. In: Proceedings of the Annual Meeting and Exhibition of the International Society for Magnetic Resonance in Medicine, Virtual; August 8-14, 2020

10. Mutsaerts HJ, Steketee RM, Heijtel DF, et al. Inter-vendor reproducibility of pseudo-continuous arterial spin labeling at 3 Tesla. PLoS One 2014;9:e104108 CrossRef Medline

11. Mutsaerts HJ, van Osch MJ, Zelaya FO, et al. Multi-vendor reliability of arterial spin labeling perfusion MRI using a near-identical sequence: implications for multi-center studies. Neuroimage 2015;113:143-52 CrossRef Medline

12. Zaharchuk G. Arterial spin labeling for acute stroke: practical considerations. Transl Stroke Res 2012;3:228-35 CrossRef Medline

13. Heijtel DF, Mutsaerts HJ, Bakker E, et al. Accuracy and precision of pseudo-continuous arterial spin labeling perfusion during baseline and hypercapnia: a head-to-head comparison with 150 H2O positron emission tomography. Neuroimage 2014;92:182-92 CrossRef Medline

14. Chen Y, Wang DJ, Detre JA. Test-retest reliability of arterial spin labeling with common labeling strategies. J Magn Reson Imaging 2011;33:940-49 CrossRef Medline

15. Gevers S, van Osch MJ, Bokkers RP, et al. Intra- and multicenter reproducibility of pulsed, continuous and pseudo-continuous arterial spin labeling methods for measuring cerebral perfusion. $J$ Cereb Blood Flow Metab 2011;31:1706-15 CrossRef Medline

16. Biagi L, Abbruzzese A, Bianchi MC, et al. Age dependence of cerebral perfusion assessed by magnetic resonance continuous arterial spin labeling. J Magn Reson Imaging 2007;25:696-702 CrossRef Medline
18. Mutsaerts HJ, Petr J, Václavů L, et al. The spatial coefficient of variation in arterial spin labeling cerebral blood flow images. J Cereb Blood Flow Metab 2017;37:3184-92 CrossRef Medline

19. Ibaraki M, Nakamura K, Toyoshima $H$, et al. Spatial coefficient of variation in pseudo-continuous arterial spin labeling cerebral blood flow images as a hemodynamic measure for cerebrovascular steno-occlusive disease: a comparative $15 \mathrm{O}$ positron emission tomography study. J Cereb Blood Flow Metab 2019;39:173-81 CrossRef Medline

20. Robertson AD, Matta G, Basile VS, et al. Temporal and spatial variances in arterial spin-labeling are inversely related to large-artery blood velocity. AJNR Am J Neuroradiol 2017;38:1555-61 CrossRef Medline

21. Mutsaerts HJ, Petr J, Bokkers RP, et al. Spatial coefficient of variation of arterial spin labeling MRI as a cerebrovascular correlate of carotid occlusive disease. PLoS One 2020;15:e229444 CrossRef Medline

22. Mutsaerts HJ, Petr J, Groot P, et al. ExploreASL: an image processing pipeline for multi-center ASL perfusion MRI studies. Neuroimage 2020;219:117031 CrossRef Medline

23. Pfefferbaum A, Chanraud S, Pitel AL, et al. Partial volume segmentation with adaptive maximum a posteriori (MAP) approach Psychiatry Res 2010;182:266-73 CrossRef Medline

24. Petr J, Mutsaerts HJ, De Vita E, et al. Effects of systematic partial volume errors on the estimation of gray matter cerebral blood flow with arterial spin labeling MRI. MAGMA 2018;31:725-34 CrossRef Medline

25. Mezue M, Segerdahl AR, Okell TW, et al. Optimization and reliability of multiple postlabeling delay pseudo-continuous arterial spin labeling during rest and stimulus-induced functional task activation. J Cereb Blood Flow Metab 2014;34:1919-27 CrossRef Medline

26. Wu $\mathrm{B}$, Lou $\mathrm{X}, \mathrm{Wu} \mathrm{X}$, et al. Intra- and interscanner reliability and reproducibility of $3 \mathrm{D}$ whole-brain pseudo-continuous arterial spinlabeling MR perfusion at 3T. J Magn Reson Imaging 2014;39:402-09 CrossRef Medline

27. Zhou J, Wilson DA, Ulatowski JA, et al. Two-compartment exchange model for perfusion quantification using arterial spin tagging. J Cereb Blood Flow Metab 2001;21:440-55 CrossRef Medline

28. Petersen ET, Lim T, Golay X. Model-free arterial spin labeling quantification approach for perfusion MRI. Magn Reson Med 2006;55:219-32 CrossRef Medline

29. Chappell MA, Woolrich MW, Petersen ET, et al. Comparing modelbased and model-free analysis methods for QUASAR arterial spin labeling perfusion quantification. Magn Reson Med 2013;69:146675 CrossRef Medline

30. Li W, Liu P, Lu H, et al. Fast measurement of blood T1 in the human carotid artery at 3T: accuracy, precision, and reproducibility. Magn Reson Med 2017;77:2296-2302 CrossRef Medline

31. Kilroy E, Apostolova L, Liu C, et al. Reliability of two-dimensional and three-dimensional pseudo-continuous arterial spin labeling perfusion MRI in elderly populations: comparison with 15o-water positron emission tomography. J Magn Reson Imaging 2014;39:93139 CrossRef Medline

32. Vidorreta M, Wang Z, Rodriguez I, et al. Comparison of $2 \mathrm{D}$ and $3 \mathrm{D}$ single-shot ASL perfusion fMRI sequences. Neuroimage 2013;66:66271 CrossRef Medline 